
\title{
Determination of Transport Parameters in Liquid Binary Lithium
}

\section{Diffusion Coefficient}

\author{
Andreas Ehrl, ${ }^{\mathrm{a},=, \mathrm{c}}$ Johannes Landesfeind, ${ }^{\mathrm{b},=, *, \mathrm{z}}$ Wolfgang A. Wall, ${ }^{\mathrm{a}}$ \\ and Hubert A. Gasteiger ${ }^{b, * *}$
}

\author{
${ }^{a}$ Institute for Computational Mechanics, Department of Mechanical Engineering, Technical University of Munich, \\ Munich, Germany \\ ${ }^{b}$ Chair of Technical Electrochemistry, Department of Chemistry and Catalysis Research Center, Technical University of \\ Munich, Munich, Germany
}

\begin{abstract}
Various numerical methods for the simulation of ion-transport in concentrated binary electrolyte solutions can be found in the literature, whereas the corresponding transport parameters are rarely discussed. In this contribution, a polarization cell consisting of two electrodes separated by a porous separator is proposed to determine the concentration dependent binary diffusion coefficient of non-aqueous electrolyte solutions. Therefore, two different electrochemical methods are extended so that they can be applied to electrolyte solutions in a porous medium. Additionally, the different methods are compared with each other by means of numerical simulations. The proposed experimental setup is used to determine the concentration dependent binary diffusion coefficient of an exemplary electrolyte, lithium perchlorate dissolved in a mixture of ethylene carbonate and diethyl carbonate, and the data are compared to those available in the literature. It will be shown that the most reliable method to determine concentration dependent binary diffusion coefficients are long-term relaxation experiments in a two-electrode cell using a porous separator.

(C) The Author(s) 2017. Published by ECS. This is an open access article distributed under the terms of the Creative Commons Attribution Non-Commercial No Derivatives 4.0 License (CC BY-NC-ND, http://creativecommons.org/licenses/by-nc-nd/4.0/), which permits non-commercial reuse, distribution, and reproduction in any medium, provided the original work is not changed in any way and is properly cited. For permission for commercial reuse, please email: oa@electrochem.org. [DOI: 10.1149/2.1131704jes] All rights reserved.

(cc) BY-NC-ND
\end{abstract}

Manuscript submitted December 22, 2016; revised manuscript received February 2, 2017. Published February 22, 2017.

Advanced numerical simulation tools are important for the understanding of existing battery systems as well as the development and the optimization of future battery systems. For such numerical simulations, accuracy and reliability are key issues and depend on appropriate physical models, boundary conditions, and, most importantly, accurately determined physico-chemical parameters. For instance, the mathematical model for binary electrolyte solutions presented by Newman and Thomas-Alyea ${ }^{1}$ is based on four different concentration dependent transport parameters, namely the conductivity $\kappa(c)$, the binary diffusion coefficient $D_{ \pm}(c)$, the transference number $t_{+}(c)$, and the thermodynamic factor or the mean molar activity coefficient $f_{ \pm}(c)$; in addition, for modeling porous battery electrodes, the concentration independent so-called tortuosity factor $\tau$ to describe the effective ionic conductivity in porous electrodes with a given morphology is required. ${ }^{2}$ While the conductivity $\kappa(c)$ can be measured using turn-key conductivity sensors, the determination of the other three concentration dependent parameters is more elaborate. Experimental methods for the determination of the transference number and the thermodynamic factor are discussed, e.g., in Ehrl et al. ${ }^{3}$ and Landesfeind et al., ${ }^{4}$ while the determination of a complete set of transport parameters can also be found in the literature. ${ }^{5,6}$ An overview of the most popular experimental techniques for the determination of binary diffusion coefficients in lithium based electrolytes is given in the following.

In Castiglione et al., ${ }^{7}$ Sethurajan et al. ${ }^{8}$ and Capiglia et al., ${ }^{9}$ pulsedfield gradient Nuclear Magnetic Resonance (NMR) is used to determine the self-diffusion coefficients of ions in an electrolyte solution, which describes the mobility of ionic species in the absence of an electrochemical potential gradient. ${ }^{10}$ In Castiglione et al., ${ }^{7}$ the selfdiffusion coefficients of all ions in an electrolyte composed of LiTFSI (lithium bis(trifluoromethanesulfonyl)imide) dissolved in the ionic liquid $\mathrm{PYR}_{14}$ TFSI (N,N-dimethyl pyrrolidinium) at a molar ratio of 1:9 were determined, whereas Capiglia et al. ${ }^{9}$ investigated $\mathrm{LiPF}_{6}$,

\footnotetext{
$=$ These authors contributed equally to this work.

*Electrochemical Society Student Member.

**Electrochemical Society Fellow.

${ }^{\mathrm{c} P r e s e n t}$ address: Velling 4, 94374 Schwarzach, Germany.

${ }^{\mathrm{z}}$ E-mail: j.landesfeind@tum.de
}

$\mathrm{LiBF}_{4}$, and $\mathrm{LiN}\left(\mathrm{C}_{2} \mathrm{~F}_{5} \mathrm{SO}_{2}\right)_{2}$ in ethylene carbonate (EC) ethyl-methyl carbonate (EMC) solvent mixture (EC:EMC at 2:8 v:v). A theoretical discussion of the experimental method is given in Price. ${ }^{11}$ For this method, the major difficulty is to relate the ionic self-diffusion coefficients determined by NMR to the binary diffusion coefficients which are generally required for numerical simulations. Another method, based on Moiré patterns, was used to determine the binary diffusion coefficient $D_{ \pm}(c)$ of $\mathrm{LiClO}_{4}$ in $\mathrm{PC}$ (propylene carbonate). ${ }^{12}$ This technique is based on the optical observation of the time-dependent relaxation of the concentration profile after two electrolyte solutions with different concentrations are brought into contact. Nishida et al. used the same technique to determine $D_{ \pm}(c)$ for $\mathrm{LiPF}_{6}$, LITFSI, and $\mathrm{LiBF}_{4}$ in PC. ${ }^{13}$ The binary diffusion coefficient can also be determined from limiting current measurements with a micro disc electrode, as was shown by $\mathrm{Xu}$ and Farrington ${ }^{14}$ for $0.1 \mathrm{M} \mathrm{LiClO}_{4}$ in PC, but this method does not allow to measure the concentration dependence of $D_{ \pm}(c)$ and can only provide an average value between the chosen salt concentration and zero (the concentration at the surface of the electrode at the limiting current).

The most popular method for the determination of the concentration dependent binary diffusion coefficient $D_{ \pm}(c)$ is the so-called restricted diffusion method introduced by Harned and French, which is based on the observation of the long-term relaxation behavior following an initially induced concentration profile. ${ }^{15}$ The applicability of the method for concentrated electrolyte solutions was demonstrated by Newman and Chapman for potassium chloride in water. ${ }^{16}$ The relaxation process can be observed by different methods. In Stewart and Newman, an optical device was used to record the relaxation of the $\mathrm{LiPF}_{6}$ concentration profile in an EC diethylcarbonate (DEC) solvent mixture (EC:DEC at 1:1 w:w). ${ }^{17}$ Limiting factors of this method are the spatial resolution of the optical measurement and the complexity of the required analysis equipment. Alternatively, the relaxation of an initially induced concentration profile can be observed indirectly via the measured potential. ${ }^{6,18-23}$ In Hiller et al., ${ }^{18}$ the concentration and temperature dependent binary diffusion coefficients of LiTFSI and LiBOB (lithium bis(oxalato) borate) in polyethylene oxide (PEO) based polymer electrolytes were determined by analyzing the long-term potential relaxation following an initial current pulse in a restricted diffusion experiment. In addition, the binary diffusion coefficients 
were also calculated based on the short-term relaxation behavior of steady-state concentration profiles. Unfortunately, the binary diffusion coefficients determined by the two methods differed significantly (by up to a factor of 6). Based on different relaxation experiments, the binary diffusion coefficient of $\mathrm{LiClO}_{4}$ in $\mathrm{PC}$ electrolyte solution soaked into a glass wool filter was determined by a numerical optimization method by Georén and Lindbergh. ${ }^{24}$ Their approach was based on a physical model including solvent effects, as introduced by Georén and Lindbergh ${ }^{24}$ and Doyle, ${ }^{25}$ whereby convective effects were neglected and the tortuosity of the glass wool filter was determined using the Bruggeman relation (the latter may introduce significant errors, as was demonstrated recently by Landesfeind et al. ${ }^{2}$ ). A similar approach based on a more elaborate optimization framework was used by Nyman et al. ${ }^{19}$ and Lundgren et al. ${ }^{26}$ for $\mathrm{LiPF}_{6}$ in EC:EMC (3:7 w:w) and in EC:DEC (1:1 w:w), respectively. In both publications, solvent effects and convective transport due to the motion of ions are included in the physical model used for the numerical optimization and, in addition, the effective ionic conductivity of the used glass microfiber filters was determined experimentally. Recently, the influence of solvent effects on the determination of transport parameters was investigated numerically by Liu and Monroe. ${ }^{27}$ Note that the experimental studies listed in this paragraph ${ }^{6,19,24,26}$ obtained the binary diffusion coefficients by fitting the complete set of transport parameters to their numerical model (i.e., $D_{ \pm}(c), t_{+}(c)$, and $\left.f_{ \pm}(c)\right)$ or rely on other transport parameters, which undoubtedly will compromise the accuracy achievable for each one of the parameters.

Although, as discussed above, there are various experimental methods for the determination of binary diffusion coefficients already available in the literature, these methods require either a conversion of selfdiffusion coefficients into binary diffusion coefficients, an additional spectroscopic technique to independently monitor concentration vs. time, or a global fit involving all concentration dependent physicochemical transport parameters. For this reason, we believe, the simplicity of the here proposed experimental setup to determine $D_{ \pm}(c)$ as a function of salt concentration, requiring no optimization framework and/or provision of additional parameters other than the tortuosity, which can be measured accurately in independent experiments, ${ }^{2}$ constitutes a valuable alternative method, particularly in view of its here demonstrated accuracy and reproducibility. In the Theoretical background section, a comprehensive introduction to the volume averaged ion-transport equations for porous media is given, which is the theoretical basis for the determination of the concentration dependent binary diffusion coefficient. In the Experimental section, the experimental procedures as well as the used materials and devices are introduced. The analytical framework for the determination of binary diffusion coefficients are summarized in the section Mathematical derivation. In the latter two sections, the experimental and the theoretical differences between a cell setup with and without porous materials are highlighted. Validity and accuracy of the introduced techniques are analyzed and compared in the section Numerical validation. Because theoretically expected transients are more obvious in simulated experiments, this section aids the interpretation of experimental data. Finally, in the Results and discussion section, the concentration dependent binary diffusion coefficient of $\mathrm{LiClO}_{4}$ in EC:DEC (1:1 w:w) obtained from our measurements is given and compared with data in the literature.

\section{Theoretical Background}

Many experimental methods for the determination of ion-transport parameters are based on analytical solutions of the component mass conservation law. For a cell consisting of a porous medium filled with an electrolyte solution, the more general case of the volume averaged mass conservation law has to be considered

$$
\varepsilon \frac{\partial c}{\partial t}-\nabla \cdot\left(\frac{\varepsilon}{\tau} D_{ \pm}(c) \nabla \mathrm{c}\right)+\nabla \cdot\left(\frac{t_{+}(c)}{z_{+} \nu_{+} \mathrm{F}} \bar{i}\right)=0
$$

as introduced, e.g., in Newman and Thomas-Alyea. ${ }^{1}$ Here, the volumetric intrinsic phase average of the concentration is denoted by $c$, the charge number of the positive ionic species by $z_{+}$, the stoichiometric coefficient of the cation by $\nu_{+}$, and the volumetric phase average of the current density by $\bar{i}$ (as usual, time is denoted by $t$ and $F$ denotes the faraday constant $(96485 \mathrm{As} / \mathrm{mol}))$. The terms volumetric intrinsic phase average and volumetric phase average result from the volume averaging approach used for the mathematical description of the porous medium. In this approach, the microscopic relations are averaged over a representative element volume to yield the macroscopic transport equations. In case of the volumetric intrinsic phase average, the averaging is performed only over the electrolyte phase within the representative element volume, whereas both the volume of the electrolyte and the solid phase within the representative element volume have to be considered in case of the volumetric phase average. The volumetric intrinsic phase average multiplied by the porosity thus corresponds to the volumetric phase average. The interested reader is referred to Bear and Bachmat ${ }^{28}$ or Landstorfer and $\mathrm{Jacob}^{29}$ for a detailed derivation of these macroscopic equations. The transport parameters used in the mass conservation law are the concentration dependent binary diffusion coefficient $D_{ \pm}(c)$ and the transference number $t_{+}(c)$. The porosity $\varepsilon$ and the tortuosity $\tau$ are parameters related to the morphology of the porous medium and are frequently used to obtain the so-called effective binary diffusion coefficient $D_{ \pm, \text {eff }}=\varepsilon \tau^{-1} D_{ \pm}$.

The conservation of current is given by

$$
\nabla \cdot \overline{\boldsymbol{i}}=0
$$

with

$$
\begin{aligned}
\overline{\boldsymbol{i}}= & -\frac{\varepsilon}{\tau} \kappa(c) \nabla \Phi+\frac{\nu}{z_{+} \nu_{+}} \frac{\mathrm{R} T}{\mathrm{~F}} \frac{\varepsilon}{\tau} \kappa(c) \\
& \times\left[1+\frac{\partial \ln f_{ \pm}(c)}{\partial \ln c}\right]\left(1-t_{+}(c)\right) \frac{1}{\mathrm{c}} \nabla c
\end{aligned}
$$

Here, $\Phi$ stands for the volumetric intrinsic phase average of the electric potential with respect to a lithium reference electrode. In addition, the concentration dependent conductivity $\kappa(c)$ and the thermodynamic factor $\left[1+\partial \ln f_{ \pm}(c) / \partial \ln c\right]$ are necessary to describe the current flow. The coefficient $v=v_{+}+\nu_{-}$is based on the stoichiometry coefficients $\nu_{+}$and $\nu_{-}$resulting from the dissociation of a binary salt in its components (e.g., $v=2$ for the typical 1:1 salts used in lithium ion batteries). The gas constant is denoted by $R(8.314 \mathrm{~J} /(\mathrm{mol} \mathrm{K}))$ and the temperature by $T$ (in units of Kelvin).

In combination with Eq. 2, Eq. 1 can be simplified to a onedimensional scalar transport equation

$$
\frac{\partial \mathrm{c}}{\partial t}-\frac{1}{\tau} D_{ \pm}\left(c_{0}\right) \frac{\partial^{2} c}{\partial x^{2}}=0
$$

with the volumetric phase average of the flux density $\bar{N}$ as the corresponding boundary condition

$$
\bar{N}=-\frac{\varepsilon}{\tau} D_{ \pm}\left(c_{0}\right) \frac{\partial c}{\partial x}+\frac{\mathrm{t}_{+}\left(c_{0}\right)}{z_{+} \nu_{+} \mathrm{F}} \bar{i}
$$

if the following assumptions are valid:

1. The experimental setup resembles an ideal geometrical configuration, as is for example satisfied for a setup consisting of two parallel and aligned flat electrodes separated by an electrolyte layer and completely enclosed by insulators. In this case, concentration and potential gradients are exclusively orientated in $\mathrm{x}$-direction (i.e., normal to the electrodes). As a result, ion-transport in the electrolyte solution can be described by a one-dimensional partial differential equation. In reality, this ideal configuration can be approximated by a two electrode cell with a large radius to distance ratio.

2. The assumption of a zero order approximation for the concentration dependent binary diffusion coefficient $\left.D_{ \pm}(c)\right|_{c_{o} \pm \delta c}=D_{ \pm}\left(c_{0}\right)$ and the transference number $\left.t_{+}(c)\right|_{c_{o} \pm \delta c}=t_{+}\left(c_{0}\right)$ has to be valid. This assumption is fulfilled if the binary diffusion coefficient and the transference number are concentration independent or if the concentration variations $\delta c$ around an initial concentration 
$c_{0}$ are small. The same condition is required for the remaining ion-transport parameters, namely the conductivity and the thermodynamic factor. Typical concentration dependent transport parameters of various binary electrolyte solutions usually fulfill this assumption, as is demonstrated in the section Numerical validation.

3. The porosity $\varepsilon$ and the tortuosity $\tau$ are constant with respect to time and space.

At the boundaries, the relation between the current density and the flux density $\bar{i}=z_{+} \nu_{+} \mathrm{F} \bar{N}$ can be used to reformulate Eq. 5 .

$$
\bar{i}=-z_{+} \nu_{+} \mathrm{F} \frac{\frac{\varepsilon}{\tau} D_{ \pm}\left(c_{0}\right)}{1-t_{+}\left(c_{0}\right)} \frac{\partial c}{\partial x}
$$

A more detailed derivation of these equations is given, e.g., by Newman and Thomas-Alyea ${ }^{1}$ or by Ehrl. ${ }^{30}$

The partial differential equation given in Eq. 4 can be solved analytically for different types of Boundary Conditions (BCs) and Initial Conditions (ICs). As a result, an expression for the concentration difference $\Delta c=c_{\mathrm{A}}-c_{C}$ between the Anode (A) and the Cathode (C) can be obtained, which for a given set of $\mathrm{BCs} / \mathrm{ICs}$ correlates $\Delta c$ with the concentration dependent binary diffusion coefficient and transference number, with the chosen salt bulk concentration, and the electrode morphology related porosity and tortuosity, in the general form Ref. 1

$$
\Delta c=c_{\mathrm{A}}-c_{C}=\mathrm{f}\left(D_{ \pm}\left(c_{0}\right), t_{+}\left(c_{0}\right), \varepsilon, \tau, \mathrm{c}_{0}\right)
$$

If the concentration difference $\Delta c$ for a specific set of $\mathrm{BCs} / \mathrm{ICs}$ and the corresponding functional description of Eq. 7 with the remaining parameters are known, it is possible to calculate the binary diffusion coefficient $D_{ \pm}(c)$. The functional description to calculate the binary diffusion coefficient from Eq. 7 with different experimental procedures is presented in the following. For polarization experiments, we further define the $\mathrm{x}$-axis of the one-dimensional coordinate system to point from the electrochemical cathode with $x_{C}=0$ toward the electrochemical anode with $x_{A}=l$, as a result of which, the concentration gradient will always be positive during the polarization experiment and the subsequent relaxation phase if $\mathrm{Li}^{+}$ions are the only reacting species in the system (i.e., the anode concentration $\mathrm{c}_{\mathrm{A}} \geq$ the cathode concentration $\mathrm{c}_{\mathrm{C}}$ ).

In this study, the concentration difference $\Delta c$ is determined indirectly by utilizing its correlation with the measured cell potential $U=\Delta \Phi+\eta$, where the potential difference between anode and cathode $\Delta \Phi=\Phi_{\mathrm{A}}-\Phi_{\mathrm{C}}$ is the volumetric intrinsic phase average of the electric potential and $\eta$ is the kinetic overpotential of the anodic and cathodic charge transfer reactions. To do so, Eq. 3 has to be reduced to its one-dimensional form as was demonstrated above for the ion-transport equation. This dimensional reduction is also only valid if the same conditions as for the ion-transport equation are fulfilled, especially the small concentration variation $\Delta c$ between anode and cathode. In the absence of current flow (i.e., $\bar{i}=0$ ) across the electrodes, the kinetic overpotential $\eta$ term is zero and the integration of Eq. 3 along a one-dimensional path directed from the cathode to the anode gives

$$
\mathrm{U}=\Delta \Phi=\frac{\nu}{z_{+} \nu_{+}} \frac{\mathrm{R} T}{\mathrm{~F}}\left[1+\frac{\partial \ln f_{ \pm}\left(c_{0}\right)}{\partial \ln c}\right]\left(1-t_{+}\left(c_{0}\right)\right) \ln \frac{c_{\mathrm{A}}}{c_{\mathrm{C}}}
$$

where the thermodynamic factor (TDF) is assumed to be constant for small concentration variations around the bulk concentration $c_{0}$, as was already assumed above for the binary diffusion coefficient and the transference number. In order to use the cell potential as a measure for the concentration difference $\Delta c$, it is necessary to introduce an additional linearization for the term $\ln c_{\mathrm{A}} / c_{\mathrm{C}}$. If the concentration difference $\Delta c=c_{\mathrm{A}}-c_{C}$ is small compared to the initial concentration $c_{0}\left(\Delta c<<c_{0}\right)$, the logarithmic term in Eq. 8 can be approximated by

$$
\ln \frac{c_{A}}{c_{\mathrm{C}}} \approx \frac{\Delta c}{c_{0}}
$$

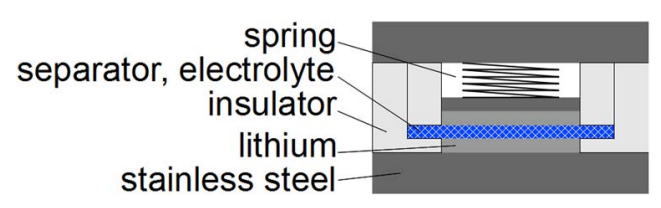

Figure 1. Schematic of two-electrode cell with symmetrical lithium electrodes and larger diameter, aligned separators, which was used for pulse experiments in the determination of binary diffusion coefficients.

as was shown, e.g., by Bruce and Vincent. ${ }^{31}$ Under these conditions, the cell potential in the absence of current flow (s. Eq. 7) will be directly proportional to the concentration difference $\Delta \mathrm{c}$ between anode and cathode

$$
U=\Delta \Phi \propto \Delta c
$$

This is one of the central aspects for the electrochemically based determination of transport parameters. The same method was also applied in, e.g., Ma et al., ${ }^{23}$ Zugmann et al., ${ }^{32}$ or Valøen et al. ${ }^{6}$

\section{Experimental}

Mixtures of ethylene carbonate (EC, $50 \%$ by weight, Sigma Aldrich, anhydrous, 99\%) and diethyl carbonate (DEC, $50 \%$ by weight, Sigma Aldrich, anhydrous, $>99 \%$ ) were used as solvents for self-prepared electrolytes containing lithium perchlorate $\left(\mathrm{LiClO}_{4}\right.$, Sigma Aldrich, 99.99\%) salt, mixed in an argon filled and temperature controlled glove box (MBraun, $25^{\circ} \mathrm{C} \pm 1^{\circ} \mathrm{C}$, water content $<0.1$ ppm, Ar 5.0, Westfalen, $99.999 \% \mathrm{Vol})$. $\mathrm{LiClO}_{4}$ concentrations ranged from 0.01 to $2 \mathrm{M}$. Metallic lithium (Rockwood Lithium, $0.45 \mathrm{~mm}$ thickness, high purity) was used as counter electrode (CE) and working electrode (WE). The binary diffusion coefficient was determined by polarization experiments in a two electrode setup as shown in Figure 1.

All cell parts were cleaned by boiling them in a mixture of ethanol and water (Millipore, Elix, $15 \mathrm{M} \Omega$ ), thoroughly rinsing them with water, followed by overnight drying at $70^{\circ} \mathrm{C}$ in a heating oven before bringing them into the glove box. Twenty circular layers of Celgard 2500 separator (porosity 55\%, thickness $25 \mu \mathrm{m}$ ) with a diameter of $20 \mathrm{~mm}$ were placed between the two lithium electrodes with a diameter of $17 \mathrm{~mm}$. A larger separator size ensured that no stray currents could flow around the porous separators through the bulk of the electrolyte. The electrode distance is determined by the thickness of the separators, which are incompressible in the pressure range induced by the mechanical spring ( $\approx 0.1 \mathrm{MPa})$. Due to the chosen setup, the geometrical distance between the electrodes $(l)$ can be adjusted accurately to small values such as $l=20.25 \mu \mathrm{m}=0.5 \mathrm{~mm}$. As a result, a large radius to distance ratio was obtained, minimizing the influence of the edge effects of the electric field and thereby fulfilling the requirement of a one-dimensional concentration and potential gradient. It is emphasized that convective effects are also suppressed using this setup with a porous separator, which is supported by experimental data: after positive and negative polarizations of the two electrodes (aligned perpendicularly to the gravitational field), identical potential relaxation transients were observed, proving that convective transport can be neglected. After the cells were sealed with PTFE gaskets, measurements were conducted in a climate chamber outside the glove box. A Biologic VMP3 potentiostat/galvanostat was used for the electrochemical measurements and cell impedances were measured in a frequency range from $200 \mathrm{kHz}$ to $1 \mathrm{~Hz}$.

Experimental procedure used in polarization cells.-Potentiostatic steady-state and galvanostatic pulse polarization experiments were conducted using the two electrode symmetrical lithium cell shown in Figure 1. While after a long potentiostatic steady-state polarization a linear concentration profile is established between the electrodes (lower panels in Figure 2), the short galvanic pulse polarization procedure is designed such that the concentrations change 

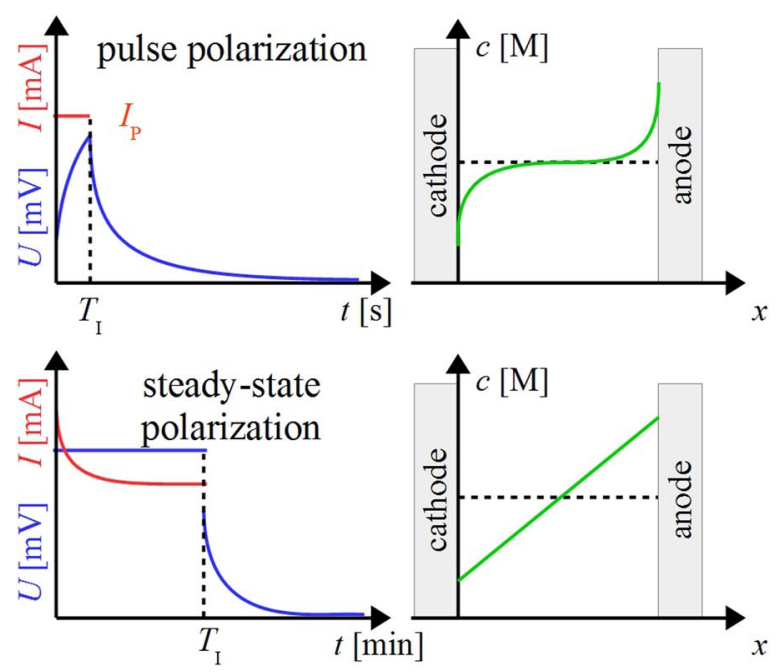

Figure 2. Schematic of the current/voltage profiles during the galvanostatic pulse polarization experiments (GPP; s. upper left panel) and of the potentiostatic steady-state polarization (SSPP; s. lower panel), together with the respective concentration profiles present at the current interruption time $T_{\mathrm{I}}$.

only in the vicinity of the electrodes (s. upper panel in Figure 2). Measurements for each salt concentration were repeated at least two times in order to check for reproducibility.

In each cell, a $6 \mathrm{~h}$ rest period was followed by several galvanostatic pulse polarization (GPP) experiments with various polarization currents $I_{\mathrm{p}}$ and times $T_{\mathrm{I}}$, whereby a pulse with a positive current flow was always followed by an identical pulse with a reversed current flow. After each individual polarization, an OCV (open circuit voltage) phase of at least $3 \mathrm{~h}$ was applied in order to ensure a complete relaxation of the concentration profile. In theory, the polarization time in a galvanostatic pulse experiment has no impact on the long-term relaxation behavior of the concentration profile. Due to the relaxation process, the requirement for small concentration differences between anode and cathode are automatically fulfilled for long times. However, due to the finite accuracy of the measurement equipment (100-200 $\mu$ V noise with the VMP3 potentiostat/galvanostat), too short polarization times with correspondingly small logarithmic cell potential variations make a determination impractical. Following these galvanostatic pulse experiments, a steady state potentiostatic polarization (SSPP) experiment was conducted. For these experiments, the polarization phase was terminated manually for each cell once the current $I(t)$ remained stable for at least 2 minutes, resulting in potentiostatic polarization times on the order of 15 to 60 minutes. The high frequency resistance of the cell was measured before and at the end of each pulse experiment to evaluate the stability of the electrolyte and was found to vary less than $3 \%$ over the course of the experiments for the nominal electrolyte concentrations of $0.5 \mathrm{M}$ to $2 \mathrm{M}$. Only for the smallest electrolyte concentration of $0.01 \mathrm{M}$, the high frequency resistance was found to decrease by $10 \%$ from the beginning to the end of the experiment $(\sim 24 \mathrm{~h})$. This decrease in the high frequency resistance implies an increase of the electrolyte conductivity, which must be due to an increase in ion concentration caused by SEI formation. Since the conductivity scales linearly with concentration at such low concentrations, the effective electrolyte concentration should be ca. $11 \mathrm{mM}$ compared to the nominal concentration of $10 \mathrm{mM}$, which is a reasonably small error, particularly since the equations for the determination of the binary diffusion coefficient are not a function of the salt concentration (shown later on by Eq. 14 and Eq. 17). Table I summarizes the applied galvanostatic pulse and steady-state potentiostatic polarization parameters. The polarization currents in galvanostatic experiments and the potentials in potentiostatic experiments were selected such that the current density would always be

Table I. Summary of the applied galvanostatic pulse polarization
(GPP) steps and the subsequent steady-state potentiostatic
polarization (SSPP) for different LiClO 4 concentrations in
EC:DEC (1:1 w:w), used to determine the binary diffusion
coefficient in the two-electrode cell setup shown in Figure 1.
Salt Concentration

GPP

below $0.3 \mathrm{~mA} / \mathrm{cm}^{2}$, as this assures the absence of lithium dendrite formation. ${ }^{33}$

\section{Mathematical Derivation}

In this contribution, two different methods for the electrochemical determination of the binary diffusion coefficient from the potential relaxation after polarization are considered. In the first method, the observed long-term relaxation behavior of the cell potential after a galvanostatic pulse (GPP) or a steady-state potentiostatic polarization experiment (SSPP) is analyzed. The second method is based on the analysis of the short-term relaxation behavior of the cell potential following a steady-state potentiostatic polarization. The first method is frequently used in the literature to determine the binary diffusion coefficient of polymer electrolytes and non-aqueous electrolyte solutions. ${ }^{6,18-23}$ However, when this method is applied to the evaluation of liquid electrolytes, most of the used experimental setups do not fulfill the ideal geometrical configuration which is required for simplification of Eq. 4. Additionally, convective effects influencing the ion transport equations in the polarization cell are not suppressed at all by these experimental setups, especially on long time scales. With regards to eliminating convective contributions to ion transport, the here proposed cell setup using porous separators is clearly superior (s. explanation in the Experimental section), but when using porous separators, the classical equations have to be adapted according to the volume averaged ion-transport equations, as will be shown in the following.

Analysis of the long-term relaxation behavior.-When using the long-term relaxation behavior for the determination of the diffusion coefficient, the two-electrode cell can be polarized by galvanostatic pulses or by potentiostatic steady-state polarization. For this method, the concentration profile $c\left(T_{\mathrm{I}}\right)$ established at the interruption time $T_{\mathrm{I}}$ is considered as the initial concentration profile. The relaxation of the concentration profile (i.e., for $\mathrm{I}=0$ ) can be observed via the relaxation of the cell potential $U(t)$. Originally, the method was developed for dilute electrolytes by Harned and French. ${ }^{15}$ The theoretical verification for concentrated solutions is given in Newman and Chapman. ${ }^{16}$ It is important to realize that in both publications only bulk electrolyte solutions, i.e., without a porous separator are considered, so that they are not valid for experiments with porous separators. However, Eq. 4 can be simply rescaled to have the same form as Eq. 1 used by Newman and Chapman, ${ }^{16}$ if we define a partial effective binary diffusion coefficient $D_{ \pm, \text {eff }}^{*}\left(c_{0}\right) \equiv \tau^{-1} \cdot D_{ \pm}\left(c_{0}\right)$ :

$$
\frac{\partial \mathrm{c}}{\partial t}-D_{ \pm, \mathrm{eff}}^{*}\left(c_{0}\right) \frac{\partial^{2} c}{\partial x^{2}}=0
$$

With this modification, the time dependent concentration at each electrode can now be determined by Eq. 2 in Newman and Chapman, ${ }^{16}$ from which $\Delta c(t)$ can be obtained by taking the difference between $c_{\mathrm{A}}(t)$ and $c_{\mathrm{C}}(t)$ (i.e., evaluating Eq. 2 in Newman and Chapman ${ }^{16}$ for cathode location $x_{\mathrm{C}}=0$ and the anode location $x_{\mathrm{A}}=l$; note that 


$$
\begin{aligned}
x \equiv y \text { and } l \equiv & a \text { in Reference 16): } \\
\Delta c(t)= & c_{A}(t)-c_{C}(t)=2 C_{1} \exp \left(-\frac{\pi^{2} D_{ \pm, \text {eff }}^{*}\left(c_{0}\right)}{l^{2}} t\right)+2 C_{3} \\
& \times \exp \left(-\frac{9 \pi^{2} D_{ \pm, \mathrm{eff}}^{*}}{l^{2}} t\right)+2 \sum_{n=3}^{\infty} C_{(2 n-1)} \\
& \times \exp \left(-\frac{(2 n-1)^{2} \pi^{2} D_{ \pm, \mathrm{eff}}^{*}\left(c_{0}\right)}{l^{2}} t\right)
\end{aligned}
$$

It is important to note that the here used partial effective binary diffusion coefficient $\left(D_{ \pm, \text {eff }}^{*}\left(c_{0}\right) \equiv \tau^{-1} \cdot D_{ \pm}\left(c_{0}\right)\right)$ must be distinguished from the commonly used effective binary diffusion coefficient $\left(D_{ \pm, \text {eff }}\left(c_{0}\right) \equiv \varepsilon \cdot \tau^{-1} \cdot D_{ \pm}\left(c_{0}\right)=\varepsilon \cdot D_{ \pm, \text {eff }}^{*}\left(c_{0}\right)\right)$, which was defined in the Theoretical background section. In the derivation of Newman and Chapman, ${ }^{16}$ a variable binary diffusion coefficient and convective effects as a result of a varying solution volume were also considered, resulting in a similar relationship as that of Eq. 12. Based on these results, the authors conclude that the variation in solution volume does not play a major role for small concentration variations. Convective effects as a result of natural or forced convection are not investigated in the latter publication, although the experimental setup uses a liquid electrolyte without porous separator, so that the absence of natural convection cannot necessarily be assumed. The different initial conditions resulting from the galvanostatic pulse and the steady-state potentiostatic polarization experiments do only have an influence on the prefactor $C_{n}$, but not on the exponential terms. A detailed derivation of this formula is given in Ehrl. ${ }^{30}$

For large times, i.e., $t \rightarrow \infty$, the first term of the analytical solution (Eq. 12) is dominating, yielding a linear relationship of the logarithmic concentration difference $\ln \Delta c(t)$ with respect to time $t$

$$
\ln \Delta c(t)=\ln \left(2 C_{1}\right)-\frac{\pi^{2} D_{ \pm, \text {eff }}^{*}\left(c_{0}\right)}{l^{2}} \cdot t \propto \ln U(t)
$$

Since the concentration difference $\Delta c$ and the measured cell potential $U$ are directly proportional (Eq. 10), the linear behavior can also be observed for the cell potential $U$ (indicated at the right-handside of Eq. 13). Thus, when plotting $\ln U(t)$ vs. $t$, the slope of the line as $t \rightarrow \infty$ (denoted as $m_{\mathrm{ln}}$ ) corresponds to $\pi^{2} \cdot D_{ \pm, \text {eff }}^{*}\left(c_{0}\right) / l^{2}$ (s. Eq. 13), so that the partial effective binary diffusion coefficient can be calculated from the linear slope $m_{\mathrm{ln}}$ :

$$
D_{ \pm, \mathrm{eff}}^{*}\left(c_{0}\right)=\frac{l^{2}}{\pi^{2}} \cdot m_{\mathrm{ln}}
$$

Advantageous in this approach is the fact that the initially imposed concentration difference by the galvanostatic pulse or the potentiostatic hold decreases with increasing time, which supports the requirement for small concentration variations introduced in the Theoretical background section.

Analysis of the short-term relaxation behavior.-In this second method, a linear concentration profile $c\left(T_{\mathrm{I}}\right)$ from a preceding potentiostatic steady-state polarization is considered as an initial concentration profile established at the time $T_{\mathrm{I}}$ when the current is interrupted, which can be approximated by a linear function for small concentration variations. It should be noted, however, that whether the linear concentration profile is obtained galvanostatically or potentiostatically is irrelevant. For the short-term relaxation from a steady-state concentration profile, the time dependent concentration difference $\Delta c(t)$ is given by the relation

$$
\Delta c(t)=\Delta \mathrm{c}\left(T_{\mathrm{I}}\right) \cdot\left(1-\sqrt{\frac{16 \cdot D_{ \pm, \mathrm{eff}}^{*}\left(c_{0}\right)}{\pi \cdot l^{2}} \cdot t}\right)
$$

showing that the short-term relaxation process is linear with respect to $\sqrt{t}$. Eq. 15 is derived by solving the partial differential equation given by Eq. 4, using a linear concentration profile as initial condition and no-flux conditions at both electrodes, which can be done by a
Laplace transformation as shown, e.g., by Ehrl. ${ }^{30}$ The same equation was presented by Hiller et al. ${ }^{18}$ for a polymer electrolyte, the only difference being that in their equation $D_{ \pm, \text {eff }}^{*}\left(c_{0}\right)$ in Eq. 15 is replaced by $D_{ \pm, \text {eff }}\left(c_{0}\right)$, as there is no porous matrix/separator. For small concentration variations, Eq. 15 can also be expressed with Eq. 8 and Eq. 9 in terms of the cell potential

$$
U(t)=U\left(T_{\mathrm{I}}\right)-U\left(T_{\mathrm{I}}\right) \frac{\sqrt{16 D_{ \pm, \mathrm{eff}}^{*}\left(c_{0}\right)}}{l \sqrt{\pi}} \sqrt{t}=U\left(T_{\mathrm{I}}\right)-m_{\mathrm{sqrt}} \sqrt{t}
$$

where $m_{\text {sqrt }}$ denotes the slope of a plot of cell potential $U$ versus $\sqrt{t}$. As a result, the partial effective binary diffusion coefficient $D_{ \pm, \text {eff }}^{*}$ can be determined by

$$
D_{ \pm, \text {eff }}^{*}\left(c_{0}\right)=\frac{\pi \cdot l^{2}}{16} \cdot\left(\frac{m_{\mathrm{sqrt}}}{U\left(T_{\mathrm{I}}\right)}\right)^{2}
$$

Here, the cell potential $U\left(T_{\mathrm{I}}\right)$ denotes the potential measured directly after current interruption.

For both methods, the tortuosity $\tau$ of the porous separator is required to determine the ultimately desired binary diffusion coefficient $D_{ \pm}\left(c_{0}\right)$, which can be determined, e.g., by the method proposed in Landesfeind et al. ${ }^{2}$ Alternatively, the effective binary diffusion coefficient $D_{ \pm, \text {eff }}\left(c_{0}\right)=\varepsilon \cdot \tau^{-1} \cdot D_{ \pm}\left(c_{0}\right)=\varepsilon \cdot D_{ \pm, \text {eff }}^{*}$ for a specific porous medium/separator can be calculated from the determined value of $D_{ \pm, \text {eff }}^{*}$, if its porosity is known. In general, careful observation of the voltage versus time behavior always gives a good indication with regards to the quality of the experimental data, also if transport parameters are determined by a numerical fitting approach.

\section{Numerical Validation}

In the following, the analytical expressions for the determination of the binary diffusion coefficient $D_{ \pm}\left(c_{0}\right)$ introduced in the Mathematical derivation section are analyzed by means of numerical simulations using typical transport parameters for lithium ion battery electrolytes and typical kinetic parameters for metallic lithium in order to evaluate their applicability for the experimental determination of binary diffusion coefficients, i.e., in order to verify the assumptions made in the Theoretical background section. All simulations are based on the Equations 1-3 and were performed with an in-house finite element research code. A detailed derivation of the used numerical methods is given in Ehrl. ${ }^{30}$

All numerical simulations are based on a one-dimensional domain representing a virtual electrode area $A=227 \mathrm{~mm}^{2}$ (corresponding to the $17 \mathrm{~mm}$ diameter lithium electrodes used in this work). The simulation and transport parameters are summarized in Table II. The chosen parameters do not represent a specific material or electrolyte but should be somehow realistic. The values given for the porosity and the tortuosity are of the same order as for the Celgard 2500 separator characterized in Landesfeind et al., ${ }^{2}$ whereas the functional description of the transport parameters is guided by the transport parameter of an electrolyte solution consisting of lithium perchlorate $\mathrm{LiClO}_{4}$ dissolved in EC:DEC (1:1 w:w) ${ }^{3,4}$ In contrast to Landesfeind et al., ${ }^{4}$ the theoretically expected Debye-Hückel behavior is included in the description of the thermodynamic factor to demonstrate that even such a strong non-linearity at small concentrations does not influence the proposed measurement method. The exchange current density $i_{0}$ in the Butler-Volmer equation is chosen to correspond to a realistic value for the kinetic resistance of a porous electrode. The remaining parameters of the Butler-Volmer equation are assumed.

Numerical analysis of the polarization experiments.-Using a quasi-1D two-electrode cell (s. Figure 1), the binary diffusion coefficient $D_{ \pm}\left(c_{0}\right)$ in this study will be determined by three different methods, which were summarized in the Mathematical derivation section. The binary diffusion coefficients $\left.D_{ \pm}\right|_{\text {long-term }} ^{\text {pulse }}$ and $\left.D_{ \pm}\right|_{\text {long-term }} ^{\text {steady-tate }}$ refer to the values calculated from the long-term relaxation behavior after a pulse and a steady-state polarization experiment, respectively (in this 
Table II. Simulation parameters for the quasi one-dimensional simulation using the finite element research code developed at the Institute for Computational Mechanics at the Technical University of Munich. The concentrations in the below given correlations are given in units of $\mathrm{mol} / \mathrm{L}$.

\begin{tabular}{|c|c|}
\hline Computational domain [mm] & $\begin{array}{c}\text { cathode at } x_{C}=0 \text { and } \\
\text { anode at } x_{A}=0.5\end{array}$ \\
\hline Virtual electrode area $A\left[\mathrm{~mm}^{2}\right]$ & 227 \\
\hline Porosity $\varepsilon[-]$ & 0.55 \\
\hline Tortuosity $\tau[-]$ & 2.6 \\
\hline Initial concentration $c_{0}[\mathrm{M}]$ & $0.01 / 1.0 / 2.0$ \\
\hline Binary diffusion coefficient $D_{ \pm}\left[\frac{\mathrm{mm}^{2}}{\mathrm{~s}}\right]$ & $2.84 \cdot 10^{-4} \exp (-0.45 \cdot c)$ \\
\hline Transference number $t_{+}[-]$ & $0.4+0.2 \cdot c-0.125 \cdot c^{2}$ \\
\hline Thermodynamic factor [-] & $1-\frac{1}{2} \cdot \frac{3.95 \cdot \sqrt{c}}{(1+63.05 \cdot \sqrt{c})^{2}}+0.907 \cdot c$ \\
\hline Conductivity $\kappa\left[\frac{\mu \mathrm{S}}{\mathrm{mm}}\right]$ & $\frac{3400 \cdot c-4700 \cdot c^{\frac{3}{2}}+2000 \cdot c^{2}}{1.0+0.2 \cdot c^{4}}$ \\
\hline \multirow{2}{*}{ Butler-Volmer equation/parameters } & $i=i_{0} \cdot\left(\frac{c}{c_{0}}\right)^{\gamma} \cdot\left[\exp \left(\frac{\alpha_{A} F}{R T} \eta\right)-\exp \left(\frac{\alpha_{C} F}{R T} \eta\right)\right]$ \\
\hline & $i_{0}=3 \frac{\mu \mathrm{A}}{\mathrm{mm}^{2}}, \quad \gamma=0, \quad \alpha_{A}=\alpha_{C}=0.5$ \\
\hline
\end{tabular}

Table III. Analytical methods for the determination of the binary diffusion coefficient $D_{ \pm}\left(c_{0}\right)$ from the three different types of experiments.

$$
\begin{aligned}
& \left.D_{ \pm}\right|_{\text {long-term }} ^{\text {pulse }},\left.D_{ \pm}\right|_{\text {long-term }} ^{\text {steady-state }} \\
& \left.D_{ \pm}\right|_{\text {short-term }} ^{\text {steady-state }}
\end{aligned}
$$$$
\frac{l^{2}}{\pi^{2}} \cdot \tau \cdot m_{\ln }
$$$$
\frac{\pi \cdot l^{2}}{16} \cdot \tau \cdot\left(\frac{m_{\mathrm{sqrt}}}{U\left(T_{\mathrm{I}}\right)}\right)^{2}
$$

work, pulse polarization experiments were done galvanostatically and steady-state polarization experiments were done potentiostatically). In addition, the binary diffusion coefficient $\left.D_{ \pm}\right|_{\text {short-term }} ^{\text {steedy-sta }}$ refers to values calculated from the short term relaxation behavior of a steadystate polarization experiment (done potentiostatically in this work). The relevant equations for determining these diffusion coefficients are given in Table III.

Figure 3 and Figure 4 depict exemplarily simulated potential relaxations after a simulated galvanostatic pulse (GPP) and steady-state

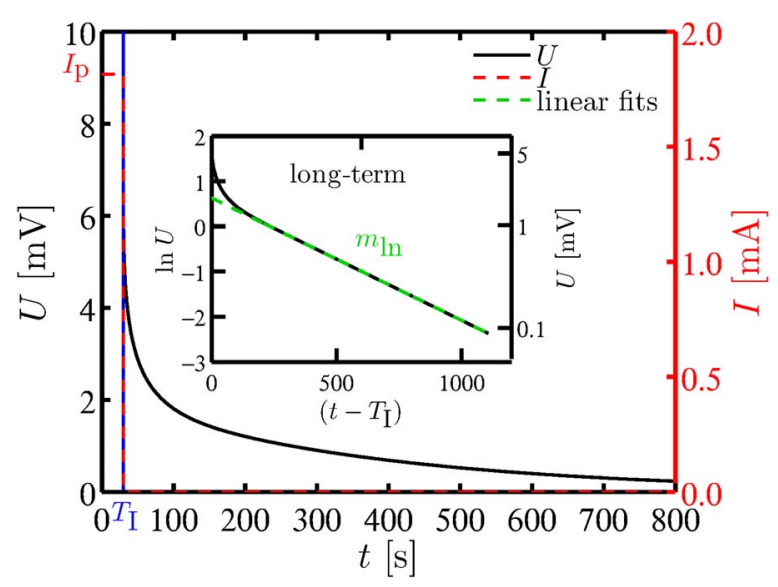

Figure 3. Simulation of a galvanostatic pulse polarization experiment (GPP) based on the parameters given in Table II and applying a polarization time of $30 \mathrm{~s}$, a polarization current density of $i_{\mathrm{p}}=0.8 \mathrm{~mA} / \mathrm{cm}^{2}$ (corresponding to a current of $I_{\mathrm{p}}=1.82 \mathrm{~mA}$ ), and an initial electrolyte concentration of $c_{0}=1 \mathrm{M}$

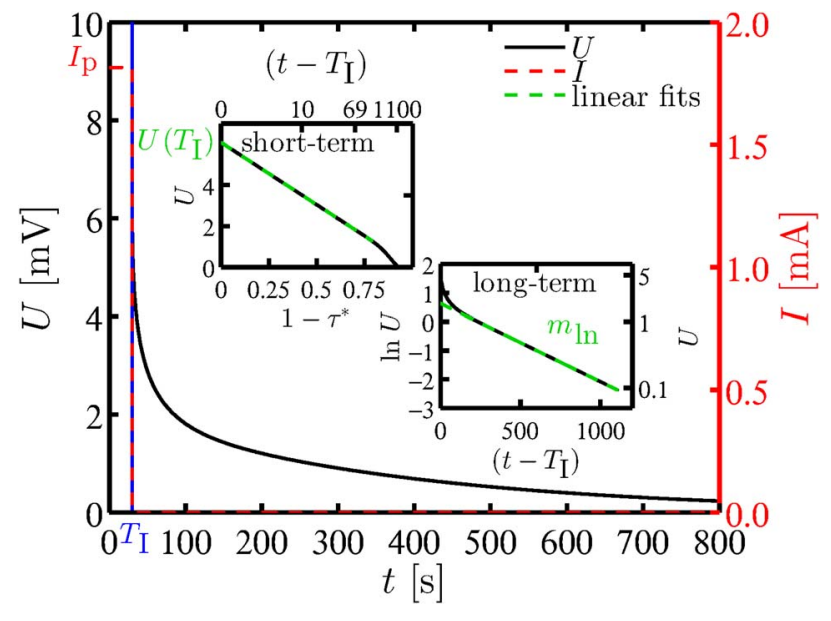

Figure 4. Plot of the relaxation phase of a simulated steady-state potentiostatic polarization experiment (SSPP) based on the parameters given in Table II, with a polarization time of $T_{\mathrm{I}}=3300 \mathrm{~s}$, a polarization potential of $U_{\mathrm{p}}=50 \mathrm{mV}$, and an initial electrolyte concentration of $c_{0}=1 \mathrm{M}$.

potentiostatic polarization (SSPP), respectively. The black and the red lines represent the numerical simulation results for the cell potential $U$ and the current $I$, respectively. The green lines stand for the simplified analytical solution presented in the section Mathematical derivation and summarized in Table III. The insets in both figures show the linear long-term relaxation of the logarithm of the cell potential vs. time with the corresponding analytical solution (Eq. 13) and the linear short-term relaxation of the cell potential with respect to the square root of time with the corresponding analytical solution (Eq. 16). In both cases, the expected time behavior predicted by Eq. 13 and Eq. 16 can be observed. The slope $m_{\mathrm{ln}}$ obtained from the long-term relaxation behavior is proportional to the partial effective binary diffusion coefficient $D_{+ \text {eff }}^{*}\left(c_{0}\right)$ as shown in Eq. 14. Similarly, the slope $m_{\text {sqrt }}$ obtained from the short-term relaxation of the cell potential allows to determine the partial effective binary diffusion coefficient as shown in Eq. 17. In this case, however, in addition to the known separator thickness $l$, the cell potential $U\left(T_{\mathrm{I}}\right)$ immediately after current interruption is necessary to calculate the partial effective binary diffusion coefficient from Eq. 17. In theory, $U\left(T_{\mathrm{I}}\right)$ is equal to the concentration overpotential resulting from the concentration difference $\Delta c$ between anode and cathode. However, in actual experiments, the cell potential $U\left(T_{\mathrm{I}}\right)$ immediately after current interruption is usually affected by additional parasitic contributions as a result of the current interruption (SEI formation currents, capacitive currents), as shown later in Figure 9 in the Results and discussion section. To overcome this commonly encountered experimental problem, the linear relation of the concentration difference $\Delta c$ (and thus of $U$ ) with respect to $\sqrt{t}$ (s. Eq. 15) can be used to extrapolate to the cell potential $U\left(T_{\mathrm{I}}\right)$, which would be solely due to the concentration overpotential immediately after current interruption (i.e., at $T_{\mathrm{I}}+\delta t$ ). This is valid, if the relaxation process of the concentration difference $\Delta c$ (diffusion process) proceeds on a slower time scale than the relaxation of potential parasitic processes as, e.g., capacitive effects. The extrapolation approach for determination of the cell potential $U\left(T_{\mathrm{I}}\right)$ at $T_{\mathrm{I}}+\delta t$ is also demonstrated in Figure 4, but as the numerical simulations do not include parasitic effects, the extrapolated and the simulated value of $U\left(T_{\mathrm{I}}\right)$ are of course identical.

Validation of determination methods.-In Figure 5, the binary diffusion coefficients determined by the different approximation equations (s. Table III) are depicted with respect to the concentration difference $\Delta c\left(T_{\mathrm{I}}\right)$ at the current interruption time, which is determined from the simulation for a bulk salt concentration of $c_{0}=1 \mathrm{M}$. In case of a pulse experiment, the concentration difference $\Delta c\left(T_{\mathrm{I}}\right)$ depends on the polarization time and the applied polarization current $I_{\mathrm{p}}$ while in 


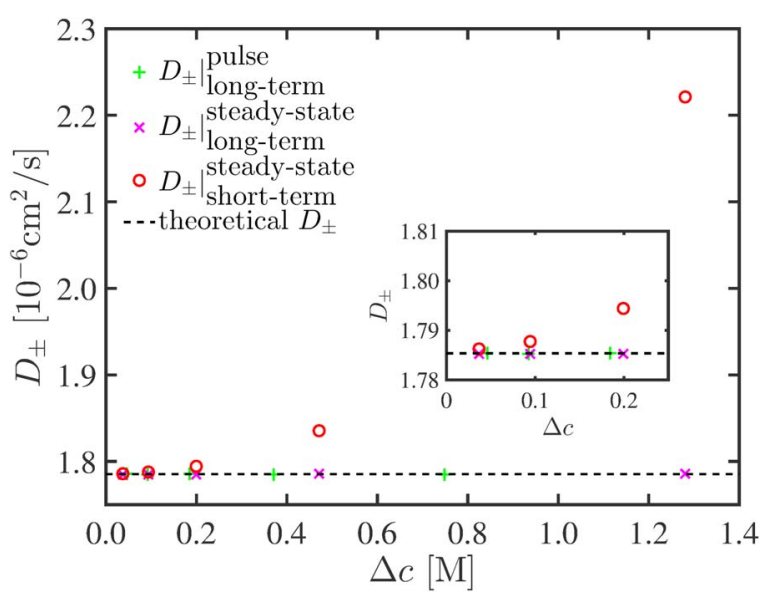

Figure 5. Binary diffusion coefficient $D_{ \pm}$with respect to the concentration difference $\Delta c\left(T_{\mathrm{I}}\right)$ between anode and cathode obtained from the simulation at the current interruption time for $c_{0}=1 \mathrm{M}\left(T_{\mathrm{I}}=300 \mathrm{~s}\right.$ for pulse experiments, see Table II for other simulation parameters).

case of a steady-state experiment it depends only on the polarization potential $U_{\mathrm{p}}$.

The results presented in Figure 5 confirm that all three evaluation methods listed in Table III are reasonably accurate up to relative concentration differences $\Delta c / c_{0}$ of $\approx 20 \%$ at the current interruption time. For the long-term relaxation behavior, the concentration difference $\Delta c\left(T_{\mathrm{I}}\right)$ at the current interruption time does not influence the quality of the obtained binary diffusion coefficient, since at the evaluated long times after the current interrupt, the concentration difference $\Delta c$ is small enough to fulfil the requirement of small concentration variations, even though this is not satisfied for short times after the current interrupt. As already mentioned earlier, this is an obvious advantage of the analysis of the long-time relaxation behavior over that of the short-time relaxation behavior. These findings are also valid for all the other bulk concentrations $c_{0}$ investigated in this study, as shown in Table IV. It may be noted here, that evaluation of the long-term relaxation behavior requires the suppression of convective effects, which is generally valid for polymer electrolytes, but in case of liquid electrolytes is only really possible when using a porous matrix (separator) containing the electrolyte rather than a pure electrolyte phase.

Compared to the long-term relaxation behavior, the results of the short-term relaxation are clearly influenced by the concentration difference $\Delta c\left(T_{\mathrm{I}}\right)$ at the current interruption time, since high values of $\Delta c\left(T_{\mathrm{I}}\right)$ violate the requirement of small concentration variations (s. Theoretical background section). Additionally, the error for the

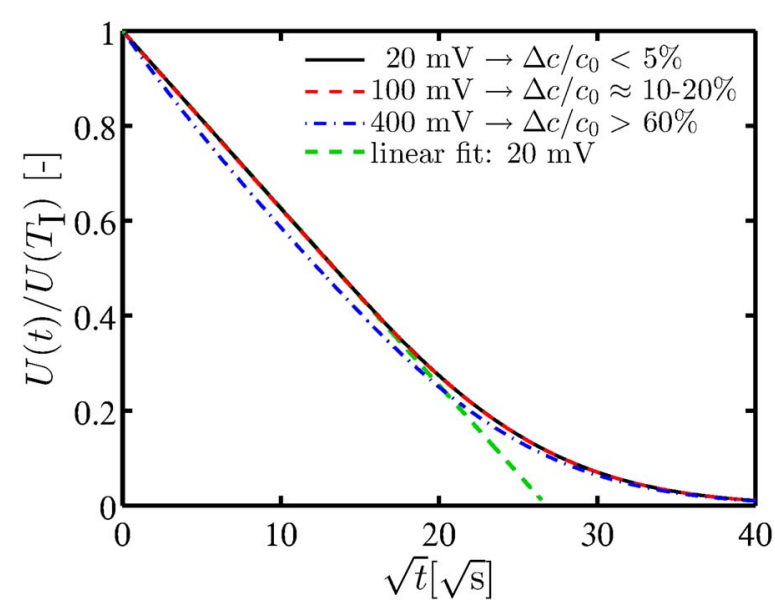

Figure 6. Relaxation behavior of the relative cell potential $U(t) / U\left(T_{\mathrm{I}}\right)$ with respect to $\sqrt{t}$ for different polarization potentials $U_{0}$ and for $c_{0}=1 \mathrm{M}$. The data are fitted in the range from $t=1 \mathrm{~s}$ to $t=10 \mathrm{~s}$ (see Table II for simulation parameters).

determined binary diffusion coefficient $\left.D_{ \pm}\right|_{\text {short-term }} ^{\text {steady-state }}$ increases for increasing bulk concentration $c_{0}$, since the absolute concentration difference $\Delta c\left(T_{I}\right)$ at current interruption is larger for a high salt concentration. The effect of the concentration difference $\Delta c\left(T_{\mathrm{I}}\right)$ on the linearity of the cell potential $U(t)$ vs. $\sqrt{t}$ is shown in Figure 6 for $c_{0}=1 \mathrm{M}$.

As already indicated in Figure 5, a concentration variation of up to $20 \%$ with respect to the salt concentration $c_{0}$ does not significantly influence the linearity of the cell potential $U(t)$ vs. $\sqrt{t}$ behavior. For a higher relative concentration difference, a deviation of the linear behavior can be observed in Figure 6 (s. blue dash-dotted line). However, the differences between the linear and a non-linear behavior of the relative potential with time is quite small, which may make it difficult to distinguish between high and low quality results, especially in real experiments with limited signal to noise ratio. Two different effects are the reason for this deviation. First, the concentration dependence of the thermodynamic factor and the transference number $t_{+}(c)$ as well as the linearization of the natural logarithm in Eq. 9 violate the assumed proportionality between the concentrations difference $\Delta c(t)$ and the cell potential $U(t)$. Additionally, the concentration dependent binary diffusion coefficient $D_{ \pm}(c)$ and transference number $t_{+}(c)$ in the ion transport equation violate the condition of constant transport parameters. This would be the only theoretical inaccuracy in case of a spectroscopic evaluation of the concentrations at cathode and anode. In case of the numerical simulation, the binary diffusion coefficient

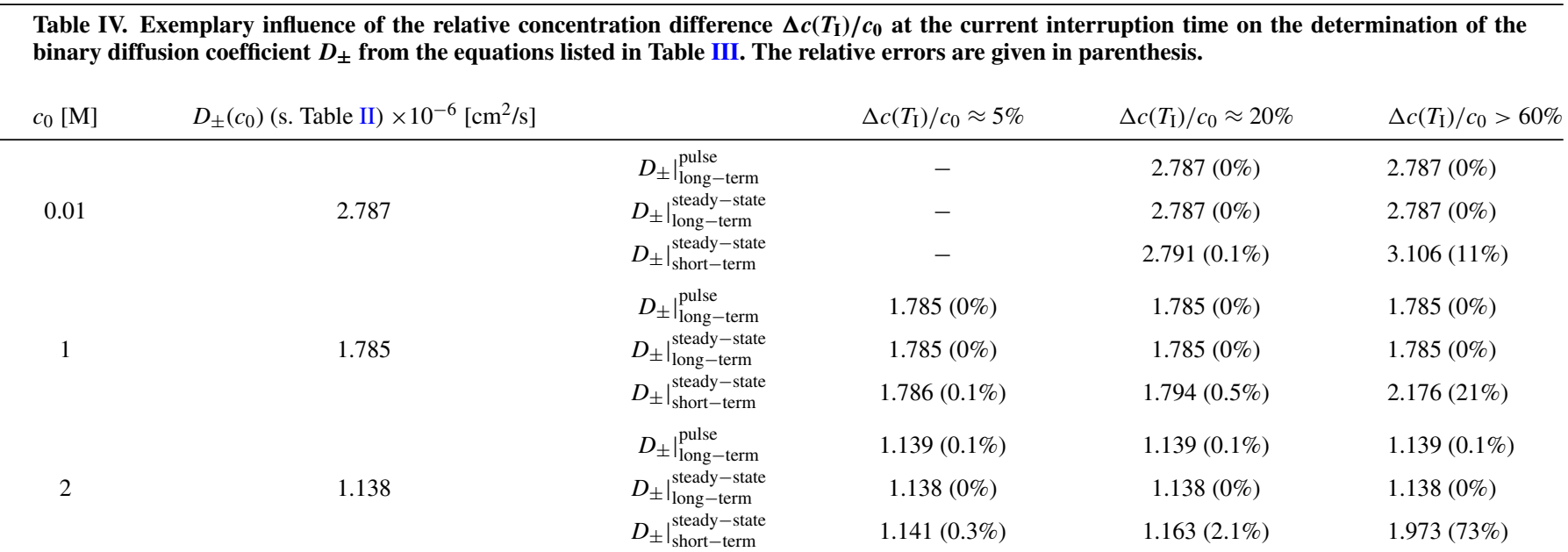


$\left.D_{ \pm}\right|_{\text {short-term }} ^{\text {steady-ste }}$ can also be determined directly from the relaxing concentration difference $\Delta c(t)$ rather than from the cell potential $U(t)$. The advantage of this approach is that potential non-linearities introduced by the concentration dependent thermodynamic factor and transference number as well as by the linearization of the natural logarithm are not included. As a result, the error in the binary diffusion coefficient $\left.D_{ \pm}\right|_{\text {short-term }} ^{\text {steady-state }}$ reduces, e.g., from $21 \%$ (s. Table IV) to $7 \%$ for a simulation of a $1 \mathrm{M}$ electrolyte solution and for the highest polarization potential $U_{\mathrm{p}}=400 \mathrm{mV}$. These values are a good indication of the errors introduced by the indirect observation of the concentration difference $\Delta c(t)$ by the cell potential $U(t)$. Besides this exemplarily estimation, a more elaborate quantification of the errors is quite complex, since the error is strongly influenced by the concentration dependence of the transport parameters which are not known a priori.

In addition to choosing a polarization potential in SSPP experiments, the polarization time is an additional experimental parameter, since a steady-state concentration profile is strictly required. If the relaxation process starts from a non-linear concentration profile, a similar non-linear behavior of the relative cell potential with respect to the square root of time as shown in Figure 6 can also be observed. In consequence, the method based on the short-term relaxation behavior requires a true linear concentration profile at the current interruption time.

As a result of these considerations, the methods based on the longterm relaxation behavior of the potential are more reliable (reflected by the smaller errors shown in Table IV), since the results are not at all influenced by the initially established concentration profile. Additionally, the requirement of small concentration variations is automatically fulfilled for the methods based on the long-term relaxation behavior. Besides, both methods observing the long-term relaxation behavior depend only on a single parameter (viz., $m_{\mathrm{ln}}$ in Eq. 14), whereas it is necessary to determine the slope and the cell potential at current interruption time for the method based on the short-term relaxation behavior (viz., $m_{\text {sqrt }}$ and $U\left(T_{1}\right)$ in Eq. 17). The effect of experimental uncertainties in these two parameters is furthermore magnified, since they appear in quadratic form in Eq. 17. Last but not least, the long-term linearity of the logarithmic potential is more distinct than the short-term linearity of the potential with respect to the square root of time, whereby it is critical to suppress convective effects at long times (s. above). This is of particular importance for experimentally determined data, which include experimental noise (the latter, of course, does not affect the numerical simulation of the experiments). Potential influences of experimental artefacts on the quality of the determination methods are discussed in detail in the next section.

\section{Results and Discussion}

In the following, the proposed methods are used to determine the partial effective binary diffusion coefficient $D_{ \pm, \text {eff }}^{*}$ of an exemplary electrolyte $\left(\mathrm{LiClO}_{4}\right.$ in EC:DEC, 1:1 w:w). The tortuosity of the here used Celgard 2500 separator $(\tau=2.5)$ which is necessary for the calculation of the binary diffusion coefficient $D_{ \pm}\left(c_{0}\right)$, is taken from Landesfeind et al., ${ }^{2}$ where it was determined from single separator layers. Because the experimental setup utilizes twenty layers of separators, it is necessary to prove the independence of the tortuosity from the number of separator layers, i.e., that the separator/separator interface does not alter the overall tortuosity of a stack of separators. Calculation of the tortuosity from the high frequency resistance measured before each pulse experiment $(20 \mathrm{Ohm})$ of a cell filled with 20 layers of the Celgard 2500 separator (porosity 55\%, active area $2.27 \mathrm{~cm}^{2}$ ) and the $0.5 \mathrm{M}$ electrolyte (conductivity $5.3 \mathrm{mS} / \mathrm{cm}$ ) yields a tortuosity of 2.65 , which is in good agreement with the value for an individual layer of the separator of 2.5. Because also contact resistances caused by, e.g., the spring in the experimental setup contribute to the high frequency resistance, we use the single layer tortuosity of 2.5 in the following analysis. As introduced in the Mathematical derivation section, the binary diffusion coefficient can be determined

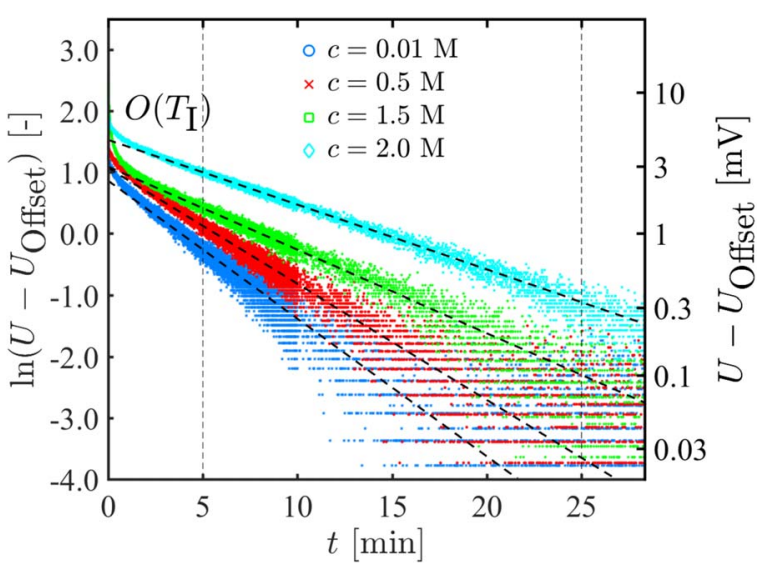

Figure 7. Experimental SSPP data (corrected for the long time offset potential) of the time relaxation of the natural logarithm of the potential $(U$ in $\mathrm{mV})$ after steady-state polarization (for polarization potentials and times see Table I), showing data for $0.01 \mathrm{M}, 0.5 \mathrm{M}, 1.5 \mathrm{M}$ and 2.0 $\mathrm{M} \mathrm{LiClO}_{4}$ in EC:DEC (1:1 w:w) obtained in the two-electrode cell described in Figure 1. The binary diffusion coefficient is determined by the linear line fits (dashed black lines) via $\left.D_{ \pm}\right|_{\text {long-term }} ^{\text {steady-state }}$ (see Eq. 14).

based on the long-term relaxation of the cell potential $U(t)$ after a pulse $\left(\left.D_{ \pm}\right|_{\text {long-term }} ^{\text {pulse }}\right.$, s. Table III) or after a steady-state polarization experiment $\left(\left.D_{ \pm}\right|_{\text {long-term }} ^{\text {steady }}\right.$, s. Table III) as well as based on the shortterm relaxation of $U(t)$ after a steady-state polarization experiment $\left(\left.D_{ \pm}\right|_{\text {short-term }} ^{\text {stedy-tate }}, \mathrm{s}\right.$. Table III). Figure 7 exemplarily shows the relaxation of the logarithm of the potential $\ln U(t)$ versus time $t$ after a steadystate polarization experiment in a $0.01 \mathrm{M}, 0.5 \mathrm{M}, 1.5 \mathrm{M}$, and $2.0 \mathrm{M}$ $\mathrm{LiClO}_{4}$ electrolyte. While the cell potential $U(t)$ relaxes to a constant value at long times, it never relaxes exactly to zero, ending at a stable open circuit potential between $-0.5 \mathrm{mV}$ and $+0.5 \mathrm{mV}$. This final value was found to be random in magnitude and sign and is attributed to changes of the lithium metal electrode surface state due to SEI formation (initiated during lithium deposition on one of the electrodes in each pulse) and roughness variation. In consequence, all relaxation curves are fitted with a free offset potential $U_{\text {Offset }}$, since the linear behavior of the open circuit potential $\ln U(t)$ with respect to time $t$ can be only observed if the open circuit potential $U(t)$ approaches zero for long times. After offset correction, the linear behavior of $\ln (U)$ vs. t can be observed consistently over times ranging from 5 to 25 minutes (marked by vertical dashed lines in Fig. 7), which allows for a quantification of the binary diffusion coefficient according to Eq. 14.

The apparent increase in noise for long times is due to the logarithmic scale, ultimately showing the digital resolution of the potentiostat. In this study, the noise level of the potential measurement was \pm 100 $\mu \mathrm{V}$. Applying the same analysis method, the binary diffusion coefficient can also be obtained from the long-term relaxation of the cell potential $U(t)$ after a pulse experiment $\left(\left.D_{ \pm}\right|_{\text {long-term }} ^{\text {pulse }}\right.$ method, s. Eq. 14) which is not shown explicitly.

The short-term relaxation of the cell potential $U(t)$ with respect to $\sqrt{t}$ after a steady-state polarization is the basis for the determination of the binary diffusion coefficient according to Eq. $17\left(\left.D_{ \pm}\right|_{\text {short-term }} ^{\text {steady-sta }}\right.$ method). Exemplary current transients during steady-state polarization experiments with an applied potential of $50 \mathrm{mV}$, i.e., the current normalized by its initial value $\left(I / I_{0}\right)$ vs. time are shown in Figure 8. In case of a $0.01 \mathrm{M}, 0.5 \mathrm{M}$, and $2.0 \mathrm{M}$ electrolyte (blue, red, and cyan lines in Figure 8), a reasonably stable current plateau was reached at the time when the current was interrupted (i.e., when switching to OCV). On the other hand, in the measurement with the $1.5 \mathrm{M}$ electrolyte (green line in Figure 8), the current starts to increase after its initial decay, which is caused by the formation of high surface area lithium and an associated decrease in the charge transfer resistance for 


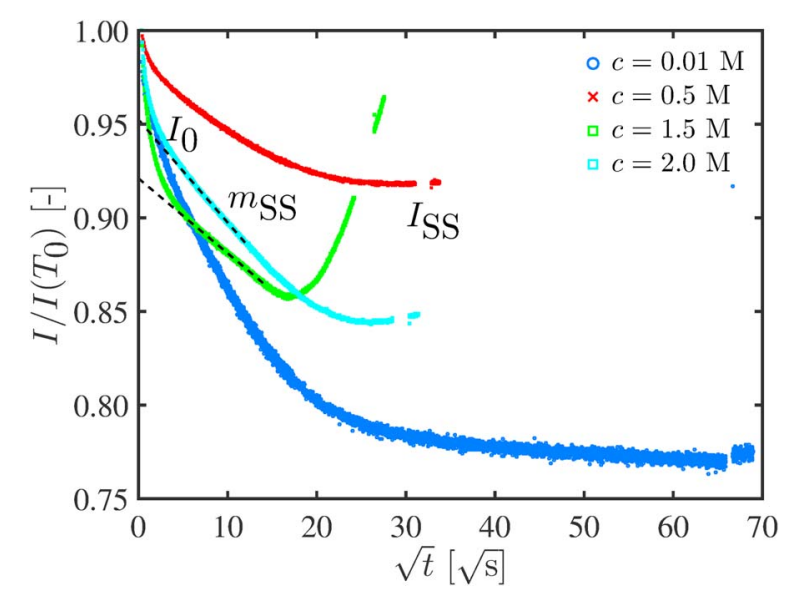

Figure 8. Exemplary steady state polarization current transients for $0.01 \mathrm{M}$, $0.5 \mathrm{M}, 1.5 \mathrm{M}$, and $2.0 \mathrm{M} \mathrm{LiClO}_{4}$ in EC:DEC (1:1 w:w) at constant polarization of $50 \mathrm{mV}$ in the two-electrode cell shown in Figure 1. The gaps in the curves toward the end of the potentiostatic polarization procedure are due to impedance measurements which were conducted during that time.

lithium dissolution/plating. In this case, a linear concentration profile at current interruption cannot be guaranteed anymore as discussed in the following.

Figure 9 depicts the corresponding OCV decays after the potentiostatic polarization phases shown in Figure 8. For the experiments with $0.5 \mathrm{M}$ and $2 \mathrm{M} \mathrm{LiClO}_{4}$ (red and light blue curves) which exhibited a steady-state current (s. Figure 8 ), a potential relaxation phase where $U(t)$ is linear vs. $\sqrt{t}$ can be clearly discerned (between 25 and $225 \mathrm{~s} / 400 \mathrm{~s}$, i.e., between ca. $5 / \sqrt{s}$ and $15 / \sqrt{s} / 20 / \sqrt{s}$ ) and is indicated by the dashed black lines in Figure 9. While the $U(t)$ vs. $\sqrt{t}$ behavior depicted in Figure 9 resembles its theoretically predicted response for $\sqrt{t}$-values exceeding $\approx 3 \mathrm{~s}^{-0.5}$ (compare Figure 9 with Figure 6), the experimental data deviate dramatically at $\sqrt{t}$-values below $\approx 3 / \sqrt{s}$, where the potential does not follow the predicted proportionality between $U\left(T_{1}\right)$ and $\sqrt{t}$. This behavior is commonly observed $^{32}$ and introduces two uncertainties in the evaluation of the diffusion coefficient based on Eq. 17: i) an ambiguity in defining the time frame over which the linear fit should be applied, which would be very straightforward if the response were to follow its theoretically predicted behavior (s. Figure 6); ii) the determination of the required

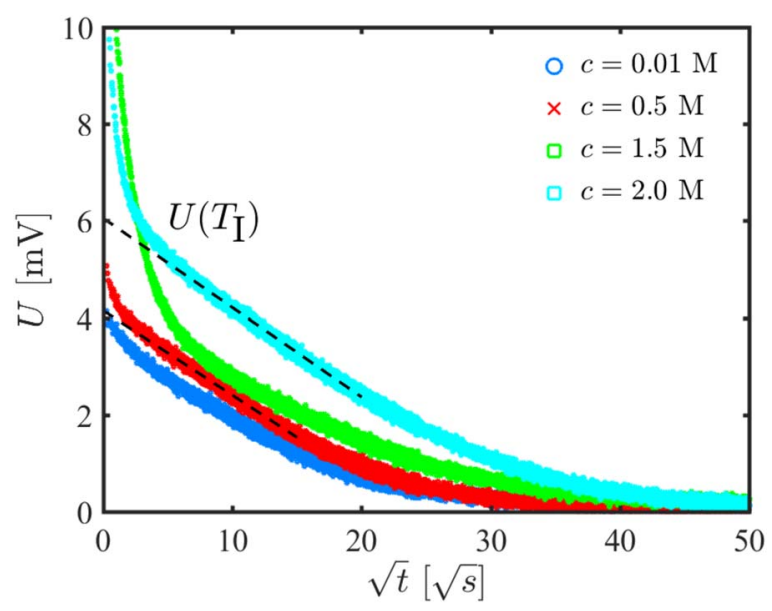

Figure 9. Short-term potential relaxation after the potentiostatic polarization experiments (SSPP) shown in Fig. 8. (0.01 M, 0.5 M, 1.5 M and 2.0 $\mathrm{M} \mathrm{LiClO}_{4}$ in EC:DEC (1:1 w:w)). The black dashed lines are a linear fit according to Eq. 16 in order to determine $\left.D_{ \pm}\right|_{\text {short-term }} ^{\text {steady-state }}$.

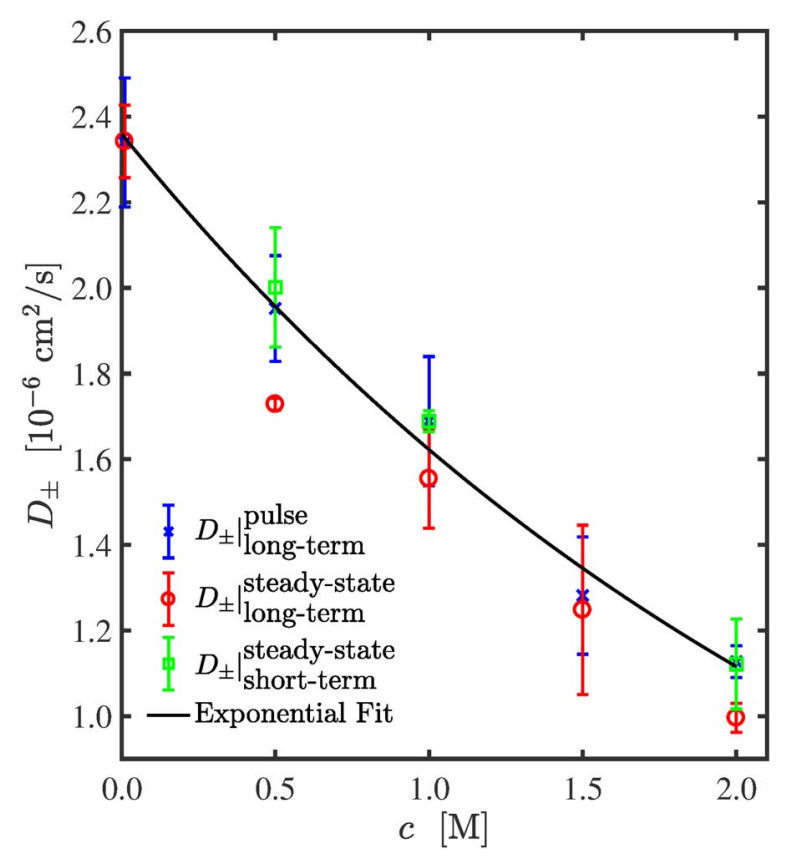

Figure 10. Concentration dependent binary diffusion coefficient $D_{ \pm}$of $\mathrm{LiClO}_{4}$ in EC:DEC $(1: 1 \mathrm{w}: \mathrm{w})$ measured in the two-electrode $\mathrm{Li}-\mathrm{Li}$ cell shown in Figure 1. The error bars represent the standard deviations from at least two different cells; the relationships to determine $D_{ \pm}$are summarized in Table III.

initial potential just after current interruption $\left(U\left(T_{1}\right)\right.$ in Eq. 17) is by no means straightforward and the value of $U\left(T_{1}\right)$ must be estimated by back-extrapolation of the linear segment to $\sqrt{t}=0$, which would be the expected voltage response in the absences of parasitic currents (s. Figure 6). This deviation of the experimental short-time response from the theoretical short-term response (i.e., below $\sqrt{t}$-values of $\approx$ $3 / \sqrt{s}$ ) is likely due to parasitic currents caused by the reformation of the SEI, particularly on the lithium electrode on which lithium plating occurred during potentiostatic polarization. Similar transients have been recorded by Odziemkowski when metallic lithium was cut while emerged in an electrolyte solution. ${ }^{34}$ In summary, determination of the binary diffusion coefficient $D_{ \pm}$from short-term relaxation experiments is clearly more difficult and error prone than from long-term relaxation experiments.

In the case where no steady-state current is obtained during potentiostatic polarization ( $\mathrm{s}$. data for $1.5 \mathrm{M} \mathrm{LiClO}_{4}$; green line in Figure 8), the conditions for an analysis of the short-term relaxation behavior are actually not fulfilled (i.e., it cannot be assumed that a steady-state linear concentration profile has been obtained). Nevertheless, the observed potential relaxation still displays a fairly linear segment (s. green line in Figure 9), so that one might be tempted to apply Eq. 17 to extract a binary diffusion coefficient. This would be clearly invalid, so that binary diffusion coefficients should only be determined from short-term relaxation experiments after having assured that a steady-state current was indeed obtained during polarization. In consequence, as the latter requirement is not met for the $1.5 \mathrm{M}$ $\mathrm{LiClO}_{4}$ experiment, our below summary of the concentration dependent binary diffusion coefficients of $\mathrm{LiClO}_{4}$ in EC:DEC (1:1 w:w) obtained by the three different analysis methods used in this study ( $\mathrm{s}$. Table III) does not include any values for the short-term relaxation experiment for the $1.5 \mathrm{M} \mathrm{LiClO}_{4}$ electrolyte. The same argumentation holds for the $0.01 \mathrm{M}$ concentration due to ambiguous linear regions during polarization in Figure 8 (s. blue line) and relaxation (s. blue line in Figure 9).

Binary diffusion coefficients determined for a $\mathrm{LiClO}_{4}$ in $\mathrm{EC}$ :DEC $(1: 1 \mathrm{w}: \mathrm{w})$ electrolyte at five concentrations using the methods summarized in Table III and plotted in Figure 10 show a good 
correlation between the three methods. The shown values are the mean values obtained from all pulses for all cells at a given concentration, and the error bars indicate their corresponding standard deviation. As already discussed in the Numerical validation section, the most reliable approach for determining the binary diffusion coefficient are the long-term relaxation methods (s. Eq. 14). Amongst these, the pulse polarization experiments $\left(\left.D_{ \pm}\right|_{\text {long-term }} ^{\text {pulse }}\right)$ have some advantage over steady-state polarization experiments $\left(\left.D_{ \pm}\right|_{\text {long-term }} ^{\text {steady-state }}\right)$ : the much shorter polarization time for pulse experiments allows for higher polarization currents which result in larger potentials during relaxation, thus leading to a better signal to noise ratio and allowing for a longer time range over which Eq. 14 can be fitted. For the methodology based on the short-term relaxation after a steady-state polarization $\left(\left.D_{ \pm}\right|_{\text {short-term }} ^{\text {steady-state }}\right)$, the generally observed parasitic currents from SEI formation and the formation of high surface area lithium can lead to a non-constant concentration profile and render the method less reliable, in addition to the risk of analyzing data from conditions where the imposed concentration gradients were too large (s. Table IV).

Figure 10 also includes an exponential fit for the concentration dependence of the binary diffusion coefficient based on the $\left.D_{ \pm}\right|_{\text {long-term }} ^{\text {pulse }}$ values, yielding

$$
D_{ \pm}(c)=2.36 \cdot 10^{-6} \exp (-0.375 c) \quad\left[\frac{\mathrm{cm}^{2}}{\mathrm{~s}}\right]
$$

where the $\mathrm{LiClO}_{4}$ salt concentration $c$ is given in units of mol/l. A decrease in the binary diffusion coefficient $D_{ \pm}(c)$ as observed in Figure 10 is expected theoretically. Upon increasing the salt concentration, ion-ion interactions become more prominent, leading to an increase of the viscosity of the electrolyte and thereby reducing the ionic mobilities. In addition, the binary diffusion coefficient $D_{ \pm}$determined by the described experimental method also includes additional factors such as the ratio between the total salt concentration and the solvent concentration, the thermodynamic factor, and potential volumetric effects as discussed, e.g., by Nyman et al. ${ }^{19}$ or Georén and Lindbergh. ${ }^{24}$ However, it is not necessary to separate these different effects from each other in order to use the determined binary diffusion coefficient in numerical simulations with a consistent physical model.

As discussed in the Introduction section, binary diffusion coefficients are reported based on rotating disc measurements, ${ }^{14}$ numerical fitting procedures, ${ }^{19,24,26}$ relaxations experiments with optical observation of the concentration gradient ${ }^{17}$ as well as based on analysis of the Moiré pattern. ${ }^{12}$ Similar trends are described by all publications, namely showing a decrease of the binary diffusion coefficient for increasing salt concentrations. Lundgren et al., ${ }^{26}$ Nyman et al., ${ }^{19}$ and Valøen et al. ${ }^{6}$ get a very similar strong concentration dependence ranging from $5.5 \cdot 10^{-6} \mathrm{~cm}^{2} / \mathrm{s}$ at infinite dilution to $0.5 \cdot 10^{-6} \mathrm{~cm}^{2} / \mathrm{s}$ at $2 \mathrm{M}$ salt concentrations for $\mathrm{LiPF}_{6}$ dissolved in similar electrolyte solutions (s. red dotted and dashed lines in Figure 11). Although Stewart and Newman also investigated a similar electrolyte solution $\left(\mathrm{LiPF}_{6}\right.$ in EC:DEC 1:1 w:w), they reported a disproportionally strong concentration dependence, based on concentration gradient measurements with an optical cell ${ }^{17}$ (s. solid red line in Figure 11). Using a numerical fitting method, Georén and Lindbergh ${ }^{24}$ and Nishikawa et al. ${ }^{12}$ determine the binary diffusion coefficient of $\mathrm{LiClO}_{4}$ in PC (s. blue dashed and blue line with asterisks in Figure 11). Particularly at low concentrations, their results compare well with our binary diffusion coefficients (s. blue solid line in Figure 11). Xu and Farrington ${ }^{14} \mathrm{ob}-$ tained an average diffusion coefficient between 0 and $0.1 \mathrm{M} \mathrm{LiClO}_{4}$ in PC using the rotating disc method (s. blue square in Figure 11). While generally larger diffusion coefficients are reported for $\mathrm{LiPF}_{6}$ compared to $\mathrm{LiClO}_{4}$ based electrolytes, similar ranges are obtained for each type, with our measurements being in good agreement with binary diffusion coefficients reported for similar electrolytes in the literature.

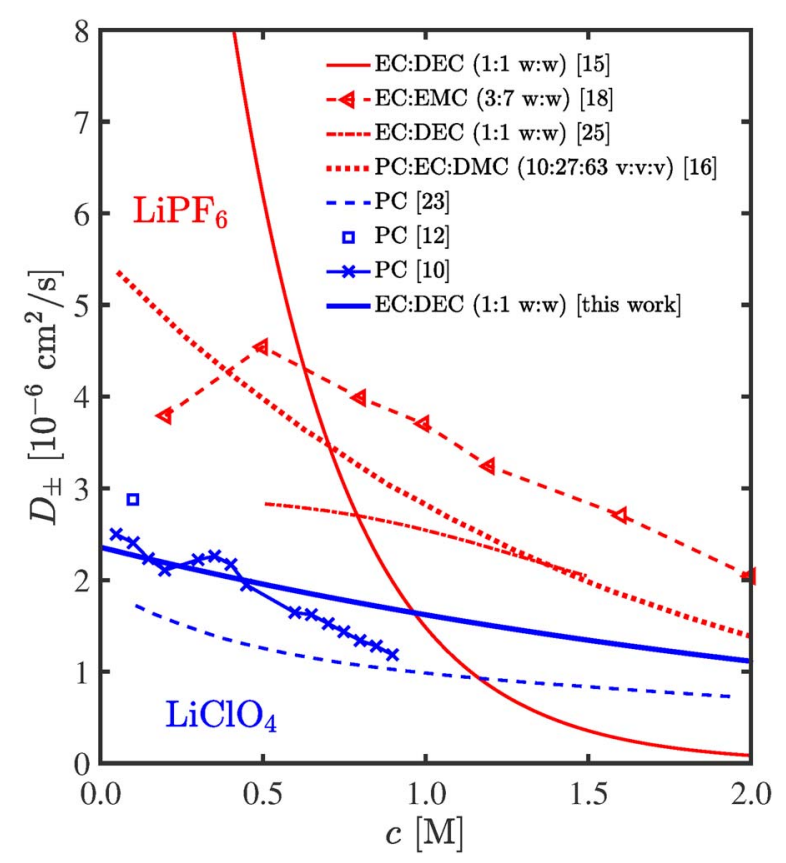

Figure 11. Literature comparison of binary diffusion coefficients for $\mathrm{LiClO}_{4}$ (blue lines/symbols) and $\mathrm{LiPF}_{6}$ (red lines/symbols) in electrolytes with different solvents with the $\mathrm{LiClO}_{4}$ electrolyte investigated in this work $\left(\mathrm{LiClO}_{4}\right.$ in EC:DEC 1:1 w:w). The literature data are: Stewart and Newman ${ }^{17}\left(\mathrm{LiPF}_{6}\right.$ in EC:DEC 1:1 w:w, RT), Nyman et al. ${ }^{19}\left(\mathrm{LiPF}_{6}\right.$ in EC:EMC, 3:7 w:w, 25 $\left.\pm 1^{\circ} \mathrm{C}\right)$, Lundgren et al. ${ }^{26}\left(\mathrm{LiPF}_{6}\right.$ in EC:DEC, 1:1 w:w, $\left.25 \pm 1^{\circ} \mathrm{C}\right)$, Valøen et al. ${ }^{6}\left(\mathrm{LiPF}_{6}\right.$ in PC:EC:DMC, 10:27:63 v:v:v, $\left.21^{\circ} \mathrm{C}\right)$, Georén and Lindbergh ${ }^{24}$ $\left(\mathrm{LiClO}_{4}\right.$ in $\left.\mathrm{PC}, \mathrm{RT}\right), \mathrm{Xu}$ and Farrington ${ }^{14}\left(\mathrm{LiClO}_{4}\right.$ in $\left.\mathrm{PC}, 25^{\circ} \mathrm{C}\right)$, Nishikawa et al. ${ }^{12}\left(\mathrm{LiClO}_{4}\right.$ in $\left.\mathrm{PC}, 25^{\circ} \mathrm{C}\right)$.

\section{Conclusions}

In this work, the concentration dependent binary diffusion coefficient is determined from the short-term and the long-term relaxation behavior after a pulse or a steady-state polarization experiment in a new two-electrode cell design using lithium metal electrodes. In contrast to similar experimental procedures available in the literature, the distance between the lithium electrodes is kept small to ensure a large radius to distance ratio and thus reduces the influence of a nonhomogenous electric field at the electrode edge. A small electrode distance is realized by 20 layers of polypropylene separators which effectively suppress convective effects which occur in experimental setups where a free electrolyte without porous matrix (separator) is used. For this new approach of using porous separators instead of free electrolyte, it was necessary to extend the analytical methods available in the literature to include the effect of the porous medium.

The accuracy of the various evaluation approaches was examined by numerical simulations, and experimental artefacts were examined by comparing simulations with the various experimental approaches using an exemplary electrolyte $\left(0.01\right.$ to $2 \mathrm{M} \mathrm{LiClO}_{4}$ in EC:DEC 1:1 $\mathrm{w}: \mathrm{w})$. The experimentally determined binary diffusion coefficients were in good agreement with those reported in the literature for similar electrolytes. Our analysis clearly shows that the most reliable and straightforward method for determining binary diffusion coefficients using a two-electrode cell is based on the long-term relaxation behavior after a galvanostatic polarization pulse using a porous separator which effectively suppresses convective effects.

\section{Acknowledgment}

We gratefully acknowledge the funding by the Bavarian Ministry of Economic Affairs and Media, Energy, and Technology for its financial support under the auspices of the EEBatt project. 


\section{List of Symbols}

\begin{tabular}{|c|c|c|}
\hline Symbol & Name & Unit \\
\hline$\kappa$ & conductivity & $\mathrm{mS} / \mathrm{cm}$ \\
\hline$D_{ \pm}$ & binary diffusion coefficient & $\mathrm{cm}^{2} / \mathrm{s}$ \\
\hline$t_{+}$ & transference number of lithium ion & - \\
\hline$f_{ \pm}$ & mean molar activity coefficient & - \\
\hline$c$ & $\begin{array}{l}\text { volumetric intrinsic phase average of the } \\
\text { concentration }\end{array}$ & $\mathrm{mmol} / \mathrm{cm}^{3}$ \\
\hline$l$ & distance between electrodes & $\mu \mathrm{m}$ \\
\hline$\varepsilon$ & porosity & - \\
\hline$t$ & time & $\mathrm{s}$ \\
\hline$\tau$ & tortuosity & - \\
\hline$z_{i}$ & ionic charge (neg./pos. for anions/cations) & - \\
\hline$v_{i}$ & stoichiometry factor & - \\
\hline $\bar{i}$ & $\begin{array}{l}\text { spatial vector of the volumetric phase average of } \\
\text { the current density }\end{array}$ & $\mathrm{A} / \mathrm{cm}^{2}$ \\
\hline $\bar{i}$ & volumetric phase average of the current density & $\mathrm{A} / \mathrm{cm}^{2}$ \\
\hline$D_{ \pm, \text {eff }}$ & effective binary diffusion coefficient & $\mathrm{cm}^{2} / \mathrm{s}$ \\
\hline$\phi$ & $\begin{array}{l}\text { volumetric intrinsic phase average of the electric } \\
\text { potential wrt. to a lithium electrode }\end{array}$ & $\mathrm{V}$ \\
\hline$U$ & cell potential & $\mathrm{V}$ \\
\hline$D_{ \pm, \text {eff }}^{*}$ & partial effective binary diffusion coefficient & $\mathrm{cm}^{2} / \mathrm{s}$ \\
\hline$m_{\mathrm{i}}$ & slopes of linear trends & various \\
\hline$A$ & electrode area & $\mathrm{cm}^{2}$ \\
\hline$I_{\mathrm{i}}$ & current & $\mathrm{mA}$ \\
\hline$R_{\mathrm{i}}$ & resistance & $\Omega$ \\
\hline$\tau^{*}$ & artificial time & - \\
\hline
\end{tabular}

1. J. Newman and K. Thomas-Alyea, Electrochemical Systems, 3rd ed., Wiley Interscience, Hoboken, (2004).
2. J. Landesfeind, J. Hattendorff, A. Ehrl, W. A. Wall, and H. A. Gasteiger, J. Electrochem. Soc., 163, A1373 (2016).

3. A. Ehrl, J. Landesfeind, H. A. Gasteiger, and W. A. Wall, in prep. (2016).

4. J. Landesfeind, A. Ehrl, M. Graf, W. A. Wall, and H. A. Gasteiger, J. Electrochem. Soc., 163, A1254 (2016).

5. A. Nyman, M. Behm, and G. Lindbergh, Electrochim. Acta, 53, 6356 (2008).

6. L. O. Valøen and J. N. Reimers, J. Electrochem. Soc., 152, A882 (2005).

7. F. Castiglione, E. Ragg, A. Mele, G. B. Appetecchi, M. Montanino, and S. Passerini, J. Phys. Chem. Lett., 2, 153 (2011).

8. A. K. Sethurajan, S. Krachkovskiy, I. C. Halalay, G. R. Goward, and B. Protas, J. Phys. Chem. B, 119, 12238 (2015).

9. C. Capiglia, Y. Saito, and H. Kageyama, J. Power Sources, 81, 859 (1999).

10. A. D. McNaught and A. Wilkinson, IUPAC. Compendium of Chemical Terminology, Blackwell Scientific Publications, Oxford, (1997).

11. W. S. Price, Concepts Magn. Reson., 9, 299 (1997).

12. K. Nishikawa, Y. Fukunaka, T. Sakka, Y. H. Ogata, and J. R. Selman, J. Electrochem. Soc., 153, A830 (2006).

13. T. Nishida, K. Nishikawa, and Y. Fukunaka, ECS Trans., 6, 1 (2008).

14. J. Xu and G. C. Farrington, J. Electrochem. Soc., 143, L44 (1996).

15. H. S. Harned and D. M. French, Ann. N. Y. Acad. Sci, 46, 267 (1945).

16. J. Newman and T. W. Chapman, AIChE J., 19, 343 (1973).

17. S. G. Stewart and J. Newman, J. Electrochem. Soc., 155, F13 (2008).

18. M. M. Hiller, M. Joost, H. J. Gores, S. Passerini, and H.-D. Wiemhöfer, Electrochim. Acta, 114, 21 (2013).

19. A. Nyman, M. Behm, and G. Lindbergh, Electrochim. Acta, 53, 6356 (2008).

20. I. V. Thorat, D. E. Stephenson, N. a. Zacharias, K. Zaghib, J. N. Harb, and D. R. Wheeler, J. Power Sources, 188, 592 (2009).

21. M. M. Doeff, L. Edman, S. E. Sloop, J. Kerr, and L. C. De Jonghe, J. Power Sources, 89, 227 (2000).

22. A. Ferry, M. Doeff, and L. DeJonghe, Electrochim. Acta, 43, 1387 (1998).

23. Y. Ma, M. Doyle, T. F. Fuller, M. M. Doeff, L. C. Jonghe, and J. Newman, J. Electrochem. Soc., 142, 1859 (1995).

24. P. Georén and G. Lindbergh, Electrochim. Acta, 47, 577 (2001).

25. C. M. Doyle, PhD Thesis, Berkeley (1996).

26. H. Lundgren, M. Behm, and G. Lindbergh, J. Electrochem. Soc., 162, 3 (2014).

27. J. Liu and C. W. Monroe, Electrochim. Acta, 167, 357 (2015).

28. J. Bear and Y. Bachmat, Introduction to Modeling of Transport Phenomena in Porous Media, Springer Netherlands, Dordrecht, (1990).

29. M. Landstorfer and T. Jacob, Chem. Soc. Rev., 42, 3234 (2013).

30. A. Ehrl, PhD Thesis, München (2016).

31. P. Bruce and C. Vincent, J. Electroanal. Chem., 225, 1 (1987).

32. S. Zugmann, M. Fleischmann, M. Amereller, R. M. Gschwind, H. D. Wiemhöfer, and H. J. Gores, Electrochim. Acta, 56, 3926 (2011).

33. D. Aurbach, E. Zinigrad, Y. Cohen, and H. Teller, Solid State Ionics, 148, 405 (2002).

34. M. Odziemkowski and D. E. Irish, J. Electrochem. Soc., 140, 1546 (1993). 